\title{
Glutamate dehydrogenase isoenzyme 3 (GDH3) of Arabidopsis thaliana is regulated by a combined effect of nitrogen and cytokinin
}

\author{
Laura Marchi ${ }^{\mathrm{a}}$, Francesca Degola ${ }^{\mathrm{a}}$, Eugenia Polverini ${ }^{\mathrm{b}}$, Thérèse Tercé-Laforgue ${ }^{\mathrm{c}}$, \\ Frédéric Dubois ${ }^{\mathrm{d}}$, Bertrand Hirel $^{\mathrm{c}}$, Francesco Maria Restivo ${ }^{\mathrm{a}, *}$ \\ ${ }^{a}$ Dipartimento di Bioscienze, Università di Parma, Parco Area delle Scienze 11/A, 43124 Parma, Italy \\ ${ }^{\mathrm{b}}$ Dipartimento di Fisica e Scienze della Terra, Università di Parma, Parco Area delle Scienze 7/A, 43124 Parma, Italy \\ ${ }^{\mathrm{c}}$ Adaptation des Plantes à leur Environnement, Unité Mixte de Recherche 1318, Institut Jean-Pierre Bourgin, Institut National de la Recherche Agronomique \\ (INRA), Centre de Versailles-Grignon, RD 10, 78026 Versailles Cedex, France \\ ${ }^{\mathrm{d}}$ Laboratoire d'Androgénèse et Biotechnologie Végétale, Université de Picardie Jules Verne, 33, Rue saint-Leu, 80039 Amiens Cedex, France
}

In higher plants, $\mathrm{NAD}(\mathrm{H})$-glutamate dehydrogenase (GDH; EC 1.4.1.2) is an abundant enzyme that exists in different isoenzymic forms. In Arabidopsis thaliana, three genes (Gdh1, Gdh2 and Gdh3) encode three different GDH subunits $(\beta, \alpha$ and $\gamma)$ that randomly associate to form a complex array of homo- and heterohexamers. The modification of the GDH isoenzyme pattern and its regulation was studied during the development of $A$. thaliana in the gdh1, gdh2 single mutants and the gdh1-2 double mutant, with particular emphasis on GDH3. Investigations showed that the GDH3 isoenzyme could not be detected in closely related Arabidopsis species. The induction and regulation of GDH3 activity in the leaves and roots was investigated following nitrogen deprivation in the presence or absence of sucrose or kinetin. These experiments indicate that GDH3 is likely to play an important role during senescence and nutrient remobilization.

\section{Keywords:}

Glutamate dehydrogenase

Isoenzymes

Subunits

Nitrogen starvation

Cytokinin

\section{Introduction}

Although the genome of Arabidopsis thaliana (Arabidopsis) is considered to be one of the most compact among land plants [1], the fact that several gene families, i.e. groups of DNA sequences sharing a high level of similarity have been maintained during evolution, remains intriguing. Therefore, in such a species a loss of redundant genes, like those encoding different isoenzymes, could be expected. However, it is generally considered that the role of duplicated genes is to provide genetic material for new biological functions whenever necessary, and thus to increase robustness against mutations [2]. Moreover, the conservation of duplicated copies of the same gene suggests that the physiological function(s) of the proteins or enzymes encoded by the corresponding gene(s) is of major importance. Comparative genomic tools are often used to shed light on the role of duplicated genes. Nevertheless, direct

Abbreviations: C, carbon; CK, cytokinins; DAS, days after sowing; GDH, NAD(H)dependent glutamate dehydrogenase; N, nitrogen.

* Corresponding author. Tel.: +39 0521 905603; fax: +39 0521905604.

E-mail address: francescomaria.restivo@unipr.it (F.M. Restivo). analysis of both duplicated gene regulation and of the physiological role of the corresponding translation products is also necessary. In Arabidopsis, the occurrence of three genes encoding $\mathrm{NAD}(\mathrm{H})$ dependent glutamate dehydrogenase (GDH; EC 1.4.1.2), an abundant and ubiquitous mitochondrial enzyme that catalyzes the reversible amination of glutamate illustrates the complexity of a redundant biological function. Moreover, although in the past few years significant progress has been made in investigations into the physiological role of GDH at the whole plant level [3], more work is required to fully understand the regulation of the synthesis of the different isoenzymes by internal and external signals during plant development.

It has been long accepted that in higher plants there are two GDH genes, Gdh1 and Gdh2, encoding the $\beta$ and $\alpha$ subunits of the enzyme respectively [3-6]. These two subunits can randomly associate to form seven distinct isoenzymes composed of $\alpha$ and $\beta$ homo- or heterohexamers depending either on the organ examined or the physiological status of the plant [3,6-10]. It has been previously established that GDH can play a major role in amino acid catabolism in plants during senescence and when nutrient remobilization is occurring [11-13]. More recently, it has been shown that in Arabidopsis a third gene encoding GDH (Gdh3) is transcribed 
and translated to form a subunit termed $\gamma$ that can assemble to form an active $6 \gamma$ oligomer GDH3 [13]. Under hydroponic growth conditions GDH3 is expressed in the roots, either at the rosette or the flowering stage. Fontaine and co-workers, also showed that the $\gamma$ subunit may be assembled into heterohexamers with subunits $\alpha$ and $\beta$, thus indicating that, at least in roots, both the biochemical properties and the physiological function of GDH need to be reexamined [14]. In this present study, the regulation of GDH3 isoenzyme activity was further examined by investigating changes in the distribution of the isoenzyme in different organs as a function of metabolic and hormonal signals. This was achieved by using both the available information in Arabidopsis gene expression databases and the results obtained when examining the changes in GDH3 activity in the leaves and roots of the gdh1 and gdh2 single or double mutants grown under different physiological conditions. The presence of GDH3 in other Arabidopsis and closely related species was also examined.

\section{Results}

\subsection{Organ-specific localization of the GDH3 isoenzyme as a function of plant development}

When Arabidopsis plants were grown on a mixture of soil and vermiculite, the $6 \gamma$ homohexamer corresponding to GDH3 was not detected following PAGE and in gel staining of leaf protein extracts of either the wild type (WT), the gdh1, gdh2 or the gdh1-2 double mutant, irrespective of the plant developmental stage (Fig. 1). Similar results were obtained when extracts of the main leaf midrib were tested for the presence of GDH3 activity (data not shown). Even in the roots of the WT and the gdh1 mutant, the GDH3 $6 \gamma$ homohexamer isoenzyme could not be detected at the three developmental stages selected for this study using plants grown on a soil-like substrate. In contrast, the presence of the GDH3 $6 \gamma$ homohexamer could be detected in the roots of gdh2 and gdh1-2 mutant plants following PAGE (Fig. 1), but only from 21 days after

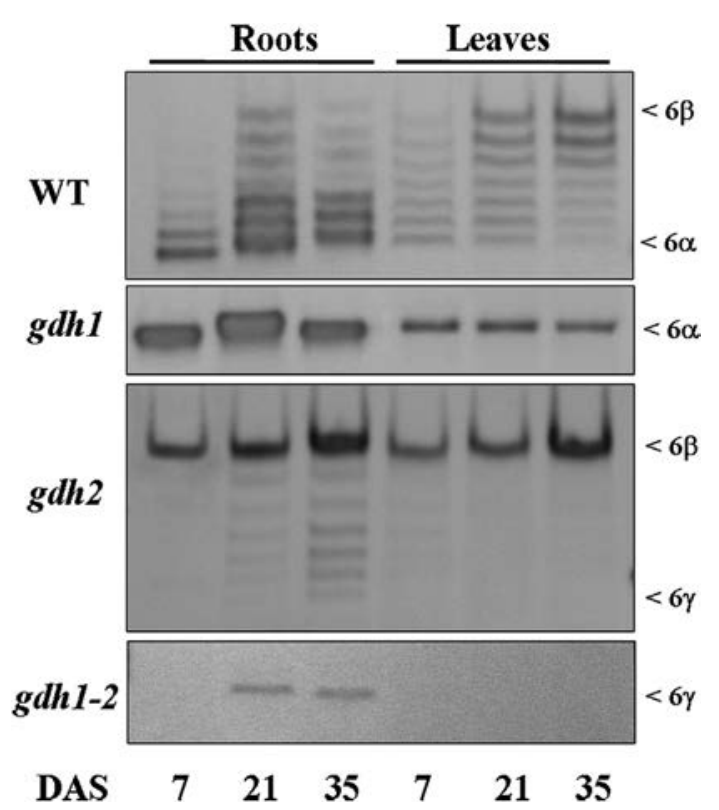

Fig. 1. Isoenzyme pattern of GDH during plant development. The leaves and roots of WT Arabidopsis and gdh1, gdh2 and gdh1-2 mutant plants were collected 7, 21 and 35 DAS. Protein extracts were subjected to native PAGE followed by NAD-GDH in gel ac-

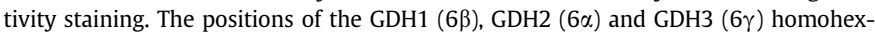
amers are indicated on the right of the gel. sowing (DAS) just before bolting and onwards. In particular in the gdh2 mutant, activity of the GDH3 $6 \gamma$ homohexamer, although not clearly visible, could be indirectly detected due to the presence of heterohexamers formed between the $\beta$ and $\gamma$ subunits. However, an activity band corresponding to the GDH3 $6 \gamma$ homohexamer could be directly identified in the gdh1-2 mutant following PAGE (Fig. 1), thus confirming the induction of synthesis of the $\gamma$ subunits of GDH3 from 21 DAS. When the different protein extracts containing the $6 \gamma$ homohexamer and the $\gamma$ and $\beta$ heterohexamers were pretreated with an antiserum raised against GDH from grapevine (see Materials and Methods Section 4.3), the GDH isoenzymes containing the $\gamma$ subunit were not detected, thus confirming that the bands visible following in gel staining for activity, corresponded to a GDH protein (Supplementary Fig. S1).

At the reproductive stage, the $6 \gamma$ homohexamer of the GDH3 isoenzyme was detected following PAGE in the closed flower buds (bd; Fig. 2A), of all the plants tested at developmental stage 10-12 but in contrast not at stage 15 , when the flowers were fully developed (fl; Fig. 2A). The $\gamma$ subunits of GDH3 were also not detected in the siliques and the flower stems, whatever their developmental stage (data not shown). Interestingly, the $\beta / \gamma$ heterohexamers found in the roots were not detected in the closed flower buds of the gdh2 mutant. In the gdh2 and gdh1-2 mutants, when the flower buds (bd) were dissected, the $6 \gamma$ homohexamer of the GDH3 isoenzyme was only visible following PAGE of stamen (st) but not pistil (pi) extracts (Fig. 2B), thus confirming there was no assembly of heterohexamers containing $\beta$ and $\gamma$ subunits. In the gdh1 mutant, there was evidence of considerably increased synthesis of the $6 \alpha$ homohexamer GDH2 isoenzyme in both the buds and flowers (Fig. 2A)

\subsection{Effect of nitrogen starvation on GDH3 activity}

The information available in the Arabidopsis microarray database (Genevestigator; https://www.genevestigator.com), indicated that nitrogen $(\mathrm{N})$ starvation induced the accumulation of $G d h 3$ gene

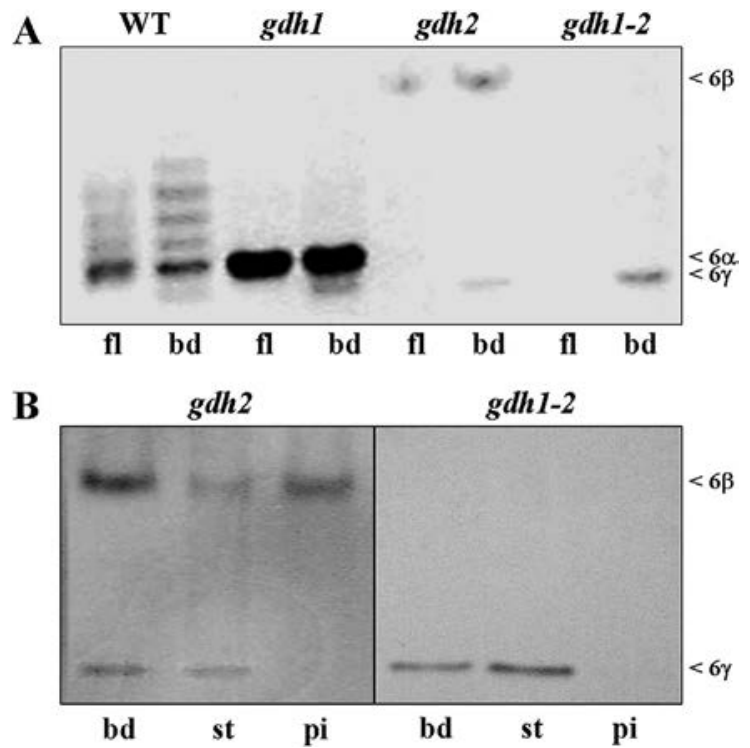

Fig. 2. Isoenzyme pattern of GDH in the reproductive organs. The different floral organs of WT Arabidopsis and gdh1, gdh2 and gdh1-2 mutant plants were collected 35 DAS. Protein extracts were subjected to native PAGE followed by NAD-GDH in gel activity staining. A) Closed flower buds (bd; stage $=10-12$ ) and open flowers (fl; stage $=15$ ): B) Closed flower buds (bd) of gdh2 and gdh1-2 mutants were used to

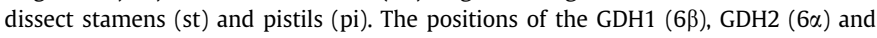
GDH3 $(6 \gamma)$ homohexamers are indicated on the right of the gel. 
transcripts, particularly in the roots of N-starved plants. When WT and gdh1-2 mutant plants grown in vitro were transferred to an $\mathrm{N}-$ free liquid medium containing sucrose, there was evidence of a time-dependent and root-specific induction of the synthesis of the $6 \gamma$ homohexamer corresponding to activity of the GDH3 isoenzyme (Fig. 3). This induction was readily visible after 2 days of $\mathrm{N}$ starvation of the gdh1-2 mutant, whereas following PAGE of root protein extracts, a very faint stained band of GDH3 activity could only be seen in the WT after 3 days of N starvation. No induction of any $\gamma$ subunits was observed in the leaves of either the gdh1-2 mutant or the WT (Fig. 3). The root-specific induction of the $6 \gamma$ homohexamer of GDH3 in the absence of $\mathrm{N}$ and in the presence of sucrose was also visible but less marked in the gdh1 mutant and confirmed by the presence of $\gamma$ and $\beta$ heterohexamers in the gdh2 mutant (data not shown). Interestingly, when the plants were transferred to an $\mathrm{N}$ free medium, the induction of the $6 \gamma$ homohexamer of GDH3 in the roots was also dependent on the availability of a carbon (C) source, since in the absence of sucrose there was no induction of GDH3 activity (Fig. 4). Similar results were obtained when sucrose was replaced by glucose, but sucrose could not be substituted by mannitol (Supplementary Fig. S2). In addition, the positive effect of soluble carbohydrates on the induction of the $6 \gamma$ homohexamer of GDH3 in the roots of the gdh1-2 mutant in the absence of N, was not modified when the plants were placed under continuous darkness (Fig. 5). In contrast, a slight induction in the synthesis of the $6 \gamma$ homohexamer occurred in the absence of sucrose when the plantlets were grown for several days under constant illumination (Fig. 5). Interestingly, when the gdh1-2 plants were grown for 12 days on an $\mathrm{N}$-free medium in the presence of sucrose, a faint band of $6 \gamma$ homohexamer GDH3 activity was detected following PAGE of leaf extracts, both under continuous illumination or continuous darkness (Fig. 5). When detached roots and leaves of gdh1-2 mutant plants were left for 6 days in an $\mathrm{N}$-free medium containing sucrose, the induction of the $6 \gamma$ homohexamer only occurred in the roots (Supplementary Fig. S3).

\subsection{Kinetin-dependent induction of GDH3 activity}

In this series of experiments, the gdh1-2 mutant, rather than the WT or the two gdh single mutants was used, as changes in the $6 \gamma$

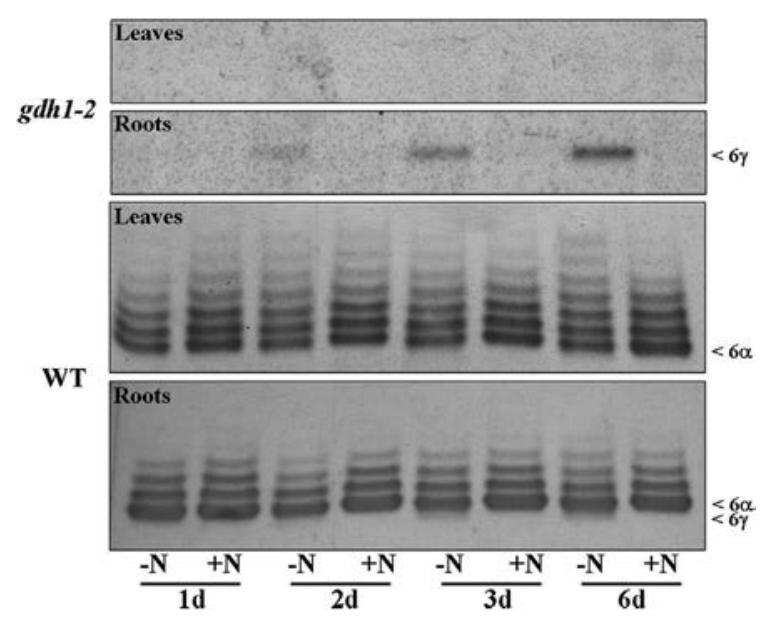

Fig. 3. Effect of nitrogen starvation on GDH3 expression. Nine day old WT Arabidopsis and gdh1-2 mutants plants were incubated in the presence $(+\mathrm{N})$ or in the absence $(-\mathrm{N})$ of inorganic $\mathrm{N}\left(4 \mathrm{mM} \mathrm{KNO}_{3}\right.$ and $\left.4 \mathrm{mM} \mathrm{NH}_{4} \mathrm{NO}_{3}\right)$ in a liquid medium containing sucrose $(1 \% \mathrm{w} / \mathrm{v})$. Root and leaf samples were collected after 1, 2, 3 and 6 days (d). Protein extracts were subjected to native PAGE followed by NAD-GDH in gel activity

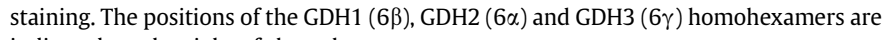
indicated on the right of the gel.

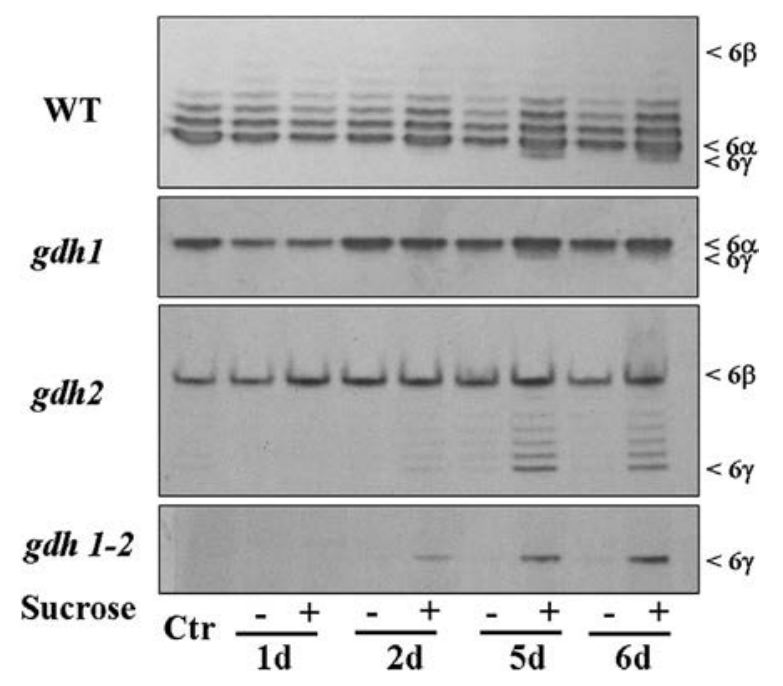

Fig. 4. Effect of carbon source on GDH3 induction in the roots of $\mathrm{N}$-starved plants. Nine days old Arabidopsis WT, gdh1, gdh2 and gdh1-2 mutant plants were incubated in Nfree liquid medium with or without sucrose $(1 \% \mathrm{w} / \mathrm{v})$. Roots were collected after 1, 2, 5 and 6 days (d). Ctr represents a control in which roots were collected from plants grown in the presence of $\mathrm{N}$ and sucrose for 6 days. Protein extracts were subjected to native PAGE followed by NAD-GDH in gel activity staining. The positions of the GDH1

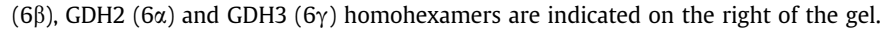

homohexamer content were more clearly visible. When gdh1-2 mutant plants were cultivated for 6 days on an $\mathrm{N}$-free liquid medium containing sucrose, the induction of root $6 \gamma$ homohexamer GDH3 activity was much higher in the presence of kinetin (Fig. 6A). Interestingly, the addition of kinetin in the presence of $\mathrm{N}$ and sucrose also induced $6 \gamma$ homohexamer activity, but to a much lower extent compared to that observed in the absence of $\mathrm{N}$. The kinetindependent induction of the $6 \gamma$ homohexamer activity of GDH3 was also observed following PAGE of leaf extracts of plants grown on an $\mathrm{N}$-free medium in the presence of sucrose.

In a second experiment, the reproductive organs of the gdh1-2 mutant including flowers, floral buds and primary and secondary flower stems were removed in order to prevent cytokinin signaling. A slight induction of the $6 \gamma$ homohexamer activity of GDH3 was observed 24 and $48 \mathrm{~h}$ after removal in the leaf midrib (Lm) but not in the leaf blade (Lb) (Fig. 6B).

\subsection{Analysis of GDH isoenzyme pattern in other Brassicaceae species}

When seven days-old plants of two Arabidopsis ecotypes (Wassilewskija and Landsberg erecta), Arabidopsis halleri and



Fig. 5. Effect of light/dark regime on GDH3 induction in the roots and leaves of $\mathrm{N}$ starved plants. Arabidopsis gdh1-2 mutant plants were incubated in an $\mathrm{N}$-free medium with or without sucrose $(1 \% \mathrm{w} / \mathrm{v})$ under constant light or in the dark. Roots and leaves were collected after 7 and 12 days (d). Protein extracts were subjected to native PAGE followed by NAD-GDH in gel activity staining. The position of the GDH3 homohexamer $(6 \gamma)$ is indicated on the right of the gel. 


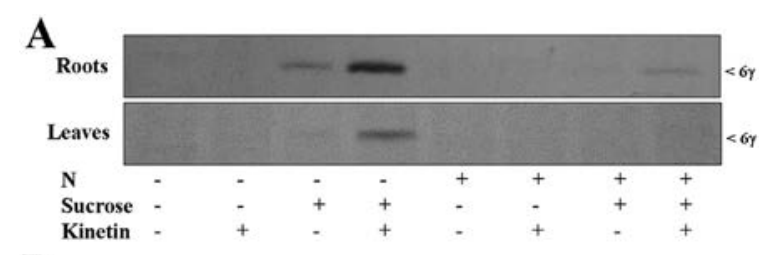

B

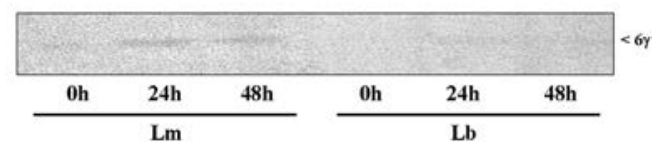

Fig. 6. Effect of cytokinin on the induction of GDH3. A) Nine days old Arabidopsis gdh1-2 mutant plants were incubated on a liquid medium with or without $\mathrm{N}$ (4 mM $\mathrm{KNO}_{3}$ and $\left.4 \mathrm{mM} \mathrm{NH} \mathrm{NO}_{3}\right)$, sucrose $(1 \% \mathrm{w} / \mathrm{v})$ and kinetin $(20 \mu \mathrm{M})$. Roots and leaves were collected after 6 days. Protein extracts were subjected to native PAGE followed by

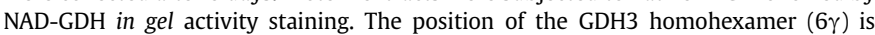
indicated on the right of the gel. B) 35 DAS, the entire flower stems of Arabidopsis gdh1-2 mutant plants were removed. 0,24 or $48 \mathrm{~h}(\mathrm{~h})$ after flower removal, leaves were sampled and the midrib (Lm) was separated from the rest of the leaf blade (Lb). Protein extracts were subjected to native PAGE followed by NAD-GDH in gel activity staining. The position of the GDH3 homohexamer $(6 \gamma)$ is indicated on the right of the gel.

Capsella rubella were grown on an $\mathrm{N}$-free liquid medium in the presence of sucrose, the $6 \gamma$ homohexamer activity of GDH3 was only induced in the roots of the Wassilewskija and Landsberg erecta A. thaliana ecotypes (Fig. 7). Moreover, when the GDH isoenzyme patterns of flower buds of various Brassicaceae species (listed in Supplementary Table S1) were analyzed following PAGE of protein extracts and activity staining: the $6 \gamma$ homohexamer activity of GDH3 was only detected in the three $A$. thaliana ecotypes (Columbia, Wassilewskija and Landsberg erecta; data not shown).

\section{Discussion}

\subsection{Developmental regulation of GDH3 expression}

The pattern of the synthesis of the $\gamma$ subunits of the GDH3 isoenzyme in Arabidopsis is more complex than that previously described by Fontaine et al. [13,14]. This could be due to the availability of $\mathrm{N}$ depending on the type of culture utilized for the experiment because these authors showed, that when Arabidopsis plants were grown in a hydroponic system in which large amounts

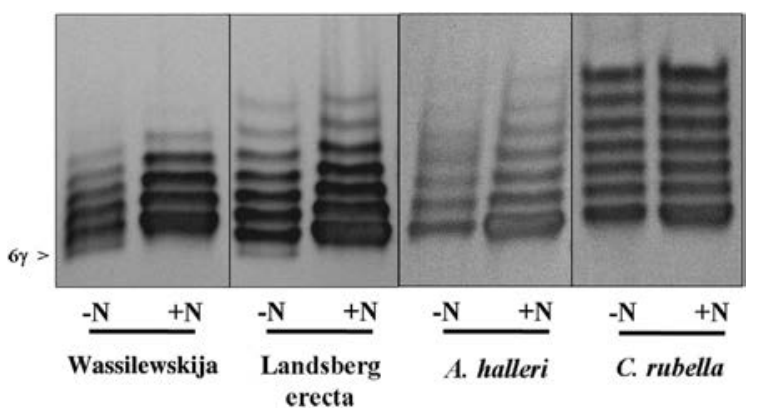

Fig. 7. Activity of GDH3 isoenzymes in different Brassicaceae species. Nine days old plants of the two Arabidopsis ecotypes Wassilewskija and Landsberg erecta, Arabidopsis halleri (A. halleri) and Capsella rubella (C. rubella) were placed on a liquid me-

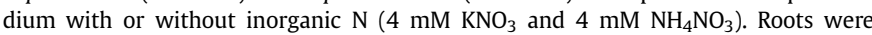
collected after 7 days. Protein extracts were subjected to native PAGE followed by NADGDH in gel activity staining. The position of the GDH3 homohexamer $(6 \gamma)$ is indicated on the left of the gel. of inorganic $\mathrm{N}$ are readily available, the GDH3 isoenzyme was preferentially expressed in the roots of plants at the rosette stage and at a very low level in floral stems. The present study, where WT, gdh1, gdh2, and gdh1-2 mutant plants were grown on a soil-like substrate, has allowed a refining of the pattern of GDH3 expression in different organs over the entire plant developmental cycle and under growth conditions closer to that of the natural environment of Arabidopsis. In particular, we observed that the $6 \gamma$ homohexamer GDH3 was induced in roots only 21 DAS, before bolting. Such pattern of GDH3 expression, different to that observed under hydroponic conditions, could be due to the timing of $\mathrm{N}$ availability in a solid substrate. This induction was clearly visible in the gdh2 and gdh1-2 mutants but was practically undetectable in the WT and the gdh1 mutant (Fig. 1). As previously shown by Fontaine et al. [13,14], these results confirm the occurrence of compensatory mechanisms controlling the GDH subunit composition depending on the presence or absence of $\alpha$ or $\beta$ and $\gamma$ subunits. For example, the $6 \gamma$ homohexamer of GDH3 is below the limit of detection in the gdh1 mutant, whereas the presence of $\beta$ and $\gamma$ heterohexamers indicates that the synthesis of the $\gamma$ subunits is up-regulated in the gdh2 mutant (Fig. 1). One can thus hypothesize that GDH2 exerts a negative control on the expression of GDH3. Moreover, it can be seen in the WT and all the mutants, that $\gamma$ subunits of GDH3 are specifically expressed in an immature male reproductive organ (stamen) of closed flower buds (Fig. 2). The finding that assembly of $\beta$ and $\gamma$ subunits was detected in the roots (Fig. 1) but not in the immature flower organ is even more intriguing. Thus, it is possible that the corresponding genes, Gdh1 and $G d h 3$, are not necessarily expressed in a particular organ or cell type, leading to cell-specific pattern of GDH isoenzyme composition. Although unknown, this regulatory control mechanism could be a way to fine-tune the enzyme activity within the plant. A time course of the expression of the three GDH isoenzymes during stamen development, combined with immunolocalization experiments could provide a more detailed picture of the temporal and spatial expression pattern of the $\gamma$ subunits of GDH3 in the male reproductive organ. Interestingly, a stamen specific expression of one of three NADH-GDH genes has also been described in rice [15].

\subsection{Nitrogen- and cytokinin-dependent regulation of GDH3 activity}

The finding that there is an induction of the synthesis of active $\gamma$ subunits of GDH3 at later stages of plant development, suggests that both in roots and stamens the isoenzyme may be involved in the recycling of both $\mathrm{C}$ and $\mathrm{N}$ assimilates during this period. This recycling could by triggered by root signals that may be translocated to the floral organs during their development. Moreover, the finding in the developing flowers that the induction of GDH3 is confined to the stamen of closed flower buds further supports this hypothesis, since pollen production and maturation, is an energy consuming process requiring large amounts of $\mathrm{C}$ and $\mathrm{N}$ assimilates $[16,17]$. Intriguingly, under standard experimental growth conditions, the synthesis of the $\gamma$ subunits of GDH3 was never detected in young seedlings or leaves, in either fully developed or senescent plants. Thus, the induction of GDH3 in the roots in Arabidopsis appears to be a root specific response depending mainly on plant development.

In order to shed light on the possible mechanisms controlling the induction of GDH3, a series of experiments were conducted to test the involvement of senescence-associated signaling systems. $\mathrm{N}$-deficiency is known to be an environmental situation that induces plant senescence [18] and $\mathrm{N}$-starved Arabidopsis plants responded to this deprivation by inducing the synthesis of active $\gamma$ subunits of GDH3 in the roots of the WT, gdh1, gdh2 and the gdh1-2 
mutants (Figs. 3 and 4). Interestingly, under these nutritional conditions GDH3 was also induced in the leaves, but with a delay as compared to the roots (Fig. 5). Altogether these observations indicate that it is an $\mathrm{N}$-starvation signal originating from the roots that is required to specifically induce GDH3 synthesis in the leaf. This situation contrasts with that found for the induction of the GDH1 and GDH2 leaf isoenzymes for which both high $\mathrm{N}$ and low sucrose are required [7-11]. In addition, sucrose feeding and an extension of the light photoperiod suggests that $\mathrm{C}$ and thus an energy source, is necessary for the induction of the synthesis of GDH3 $\gamma$ subunits both in roots and leaves under $\mathrm{N}$-limiting conditions (Figs. 4 and 5). These results fit in with the occurrence of various metabolic and biochemical regulatory control mechanisms that constantly monitors the plant nutritional status to cope with any imbalance in the $\mathrm{C}$ and $\mathrm{N}$ ratio [19-21]. For example, a local signaling mechanism depending on $\mathrm{N}$ availability could be involved - even if the presence of a parallel systemic signaling mechanism cannot be excluded - since an induction of GDH3 was observed in detached roots incubated under $\mathrm{N}$-starvation conditions (Supplementary Fig. S3).

Many plant growth and development processes are controlled by cytokinins (CK), a class of hormones that plays a major regulatory role in the control of a large repertoire of biological processes such as plant senescence, nutrient mobilization and stress responses [22]. For $\mathrm{N}$ metabolism, a specific role for CK as part of a systemic signaling process has been previously proposed [23,24]. It was demonstrated that $\mathrm{CK}$ regulate the expression of several genes involved in both $\mathrm{N}$ uptake and $\mathrm{N}$ assimilation [24,25]. Moreover some authors have proposed that $\mathrm{CK}$ are able to sense the plant nutrient status [22]. Experimental evidence is provided here that kinetin amplifies the effect of $\mathrm{N}$ starvation on the induction of GDH3. Moreover, we showed that Kinetin could also induce GDH3 activity in the roots of $\mathrm{N}$-fed plants, even if this induction was lower compared to that observed in N-starved plants. However, GDH3 induction by Kinetin was completely abolished when sucrose was omitted from the medium, regardless of $\mathrm{N}$ availability. This finding also highlight the importance of the of $\mathrm{C} / \mathrm{N}$ ratio as being part of a major check point in the signaling mechanism triggering the induction of GDH3 activity. Under standard growth conditions, CK are known to inhibit nitrate and ammonium ion uptake in roots $[22,26]$. It is therefore possible that the external application of CK could simulate $\mathrm{N}$-starvation conditions in the roots, by restricting inorganic $\mathrm{N}$ uptake and thus inducing GDH3 activity. In order to check the possible interaction between $\mathrm{N}$ and $\mathrm{CK}$ signaling in the control of GDH3 activity, it will be interesting to study its induction in mutants deficient for the $\mathrm{N}$ assimilatory and CK biosynthesis pathways.

However, if the main role of GDH is to degrade glutamate to form 2-oxoglutarate, which may be used as a respiratory substrate [13], then as in natural senescence $\mathrm{N}$ deficiency requires a supply of energy to recycle and export $\mathrm{C}$ and $\mathrm{N}$ assimilates to ensure plant reproduction. This biological response of the plant would be in line with the finding that the root mediated $\mathrm{N}$-starvation response, is induced before that occurring in the leaf (Fig. 5). Additional indirect evidence of the involvement of CK was obtained in the experiments in which the removal of the reproductive organs caused a small induction in GDH3 activity (Fig. 6B), in agreement with the transcript data of Igarashi et al. [27]. Moreover, the present study of kinetin-stimulated GDH3 enzyme activity supports the results of Igarashi et al. [27] that Gdh3 transcripts accumulated in the midribs of excised leaves, following trans-zeatin treatment. Although further research is required to indentify the regulatory elements controlling the $\mathrm{CK}$-mediated changes in $\mathrm{N}$ assimilation and recycling in relation to $\mathrm{C}$ and $\mathrm{N}$ assimilate management during the reproductive phase, it is likely that GDH3 plays a specific metabolic and regulatory function in Arabidopsis during this process.

\subsection{GDH3 expression in Brassicaceae}

If the induction of the synthesis of active $\gamma$ subunits of GDH3 is part of a general plant response to natural or induced nutrient remobilization in Arabidopsis, it might be expected that the relevant biological function would occur in related species. Somewhat surprisingly, we found little evidence for this, as the presence of the $6 \gamma$ structure of GDH3 was only detected in the two additional Arabidopsis ecotypes analysed (Fig. 7) Although A. halleri and C. rubella possess what is considered as an ancestral karyotype $(n=8)$ of Arabidopsis [28], active $\gamma$ subunits of GDH3 were not detected. It is therefore likely that following rearrangements of their genomes during evolution a functional Gdh3 gene was lost. Whether this loss is related to a specific ecophysiological adaptation of Arabidopsis to particular trophic conditions remains to be demonstrated, since the majority of the Brassicaceae species (Table S1) examined so far did not exhibit any GDH3 activity even under N-deficient conditions.

\section{Materials and methods}

\subsection{Plant material and growth conditions}

A. thaliana L. accessions and the different Brassicaceae species (Supplementary Table S1) were grown in plastic pots (5 and $10 \mathrm{~cm}$ diameter for Arabidopsis and the different Brassicaceae, respectively) containing an autoclaved $\left(121^{\circ} \mathrm{C} ; 20 \mathrm{~min}\right) 3 / 1$ mixture of soil/vermiculite purchased from a local dealer (Consorzio Agrario, Parma, Italy). Plants were fertilized weekly with $10 \mathrm{ml}$ of a diluted (1:10) solution of Murashige-Skoog (MS) medium (MS salts; Duchefa; \#M0222, Haarlem, The Netherlands). Plants were placed in a growth chamber at $25{ }^{\circ} \mathrm{C}$ under $50-70 \%$ relative humidity. Day length was $16 \mathrm{~h}$ and the light intensity was $200 \mu \mathrm{mol}$ photons $\mathrm{m}^{-2} \mathrm{~s}^{-1}$. Watering with demineralized water was performed, when required to maintain soil moisture. Prior to sowing, seeds were surface sterilized for $20 \mathrm{~min}$ in a diluted bleach solution (final concentration $=0.5 \% \mathrm{v} / \mathrm{v}$ Sodium Hypochlorite) containing $0.1 \%(\mathrm{v} / \mathrm{v})$ Tween 20 . The seeds were then washed three times with sterile demineralized water and stratified for two days in the dark at $4{ }^{\circ} \mathrm{C}$. Plant (A. thaliana $\mathrm{L}$, ecotype Columbia was mostly used for developmental and nutritional studies) samples were harvested at various time of plant development spanning from 7 DAS to the stage for which flowers were fully developed (35 DAS). At the various times of plant development, the different organs were harvested, weighed, frozen in liquid $\mathrm{N}$ and stored at $-80{ }^{\circ} \mathrm{C}$. Residual soil particles were removed from the roots following several washes with demineralized water. Roots were then dried on filter paper before freezing in liquid $\mathrm{N}$ and storage at $-80^{\circ} \mathrm{C}$. Floral stems were harvested after flower removal; 1 ) when floral buds were still closed, 2) when 3-5 flowers were fully opened and 3) when developed siliques were formed. Floral buds were composed of the entire floral meristem containing either stage 10-12 closed buds or stage 15 open buds from primary and secondary inflorescences. The different stages of flower development were defined according to those reported on the Arabidopsis electronic fluorescent protein browser; (http://bar.utoronto.ca/efp_arabidopsis/cgi-bin/efpWeb. cgi). In order to collect $A$. halleri inflorescences, 28-35 days-old plants were incubated for two weeks in a cold room at $4{ }^{\circ} \mathrm{C}$ with light provided by fluorescent tubes. At the end of this vernalization period, plants were then transferred to a controlled environment chamber using the same growth conditions as those used for $A$. thaliana. 
To study the nutritional effect on the induction of GDH3 activity, plants were grown in vitro in liquid culture. Stratified seeds were germinated in Petri dishes on 1/2 Murashige-Skoog (MS) agar medium; (MS salts; Duchefa; \#M0222, Haarlem, The Netherlands) containing $2 \%(\mathrm{w} / \mathrm{v})$ sucrose and solidified with $(0.8 \%(\mathrm{w} / \mathrm{v})$ agar) and grown for 7 days at $25{ }^{\circ} \mathrm{C}$ with a $16 \mathrm{~h} / 8 \mathrm{~h}$ photoperiod $\left(150 \mu \mathrm{mol}\right.$ photons $\left.\mathrm{m}^{-2} \mathrm{~s}^{-1}\right)$. Plants were then removed from the solid medium and transferred to Petri dishes filled with $10 \mathrm{ml}$ of $1 / 5$ diluted MS medium containing 1\% sucrose (adaptation to liquid culture condition) without shaking. After 2 days plants were quickly washed with sterile demineralized water and transferred to the $\mathrm{N}$-starvation medium (1/5 MS salts deprived of $\mathrm{KNO}_{3}$ and $\mathrm{NH}_{4} \mathrm{NO}_{3}$ ) for up to 12 days. Dark growth conditions were obtained by wrapping the plates with aluminum foil. For sample analysis, plants were removed from the Petri dishes and treated as described for the plants grown on soil.

\subsection{Protein extraction and in gel GDH activity staining}

Proteins were extracted from different tissues as described previously [6,8]. Briefly, $200 \mathrm{mg}$ of plant tissue was homogenized in $200 \mu \mathrm{l}$ of extraction buffer composed of $100 \mathrm{mM}$ Tricine ( $\mathrm{pH} 8.0$ ), $10 \mathrm{mM} \mathrm{MgSO}_{4}, 0.2 \%(\mathrm{v} / \mathrm{v}) \beta$-mercaptoethanol, $0.5 \mathrm{mM}$ PMSF, $40 \mathrm{mM} \mathrm{CaCl}_{2}, 0.5 \%(\mathrm{w} / \mathrm{v})$ polyvinylpyrrolidone, $1 \mathrm{mM}$ EDTA and $0.05 \%(\mathrm{v} / \mathrm{v})$ Triton X-100 in a $1.5 \mathrm{ml}$ Eppendorf tube using a micropestle. After two rounds of centrifugation at $15,000 \times \mathrm{g}$ for $20 \mathrm{~min}$ at $4{ }^{\circ} \mathrm{C}$, the resulting supernatant $(10 \mu \mathrm{l})$ was used for GDH in gel activity detection. In gel NAD-GDH activity detection was performed as described by Loulakakis and Roubelakis-Angelakis [29], with minor modifications. The GDH activity staining solution, containing $100 \mathrm{mM}$ Tris- $\mathrm{HCl}$ ( $\mathrm{pH} 8.8$ ), $53 \mathrm{mM}$ sodium glutamate, $0.7 \mathrm{mM}$ NAD, $0.03 \mathrm{mM}$ phenazine methosulphate and $0.3 \mathrm{mM}$ Nitro Blue Tetrazolium (NBT) was supplemented with agarose (BioRad, Hercules, CA, USA) at a final concentration of $0.4 \%(\mathrm{w} / \mathrm{v})$ and poured onto the gel. Enzyme activity staining was performed at $37^{\circ} \mathrm{C}$ in the dark and stopped by replacing the staining solution with distilled water. Photographs of the gel were taken with a Kodak EDAS120 digital camera (Eastman Kodak Company, Rochester, NY, USA) and the activity of the different GDH isoenzymes was quantified by the 1D image analysis software provided by the manufacturer. Both PAGE and in gel GDH activity detection were performed at least in triplicate with different plants for each experiment. Comparable results were obtained in each replicate.

\subsection{GDH immunoinactivation assay}

To test the GDH specificity of in gel GDH activity staining procedure, protein extracts were pre-incubated with a specific antiGDH antiserum before loading on the gel. The GDH antiserum $(0.15 \mu \mathrm{l})$ raised against grapevine GDH [29] was incubated in ice with $20 \mu \mathrm{l}$ of plant protein extracts for $1 \mathrm{~h}$. Before loading on the gels the incubation mixture was centrifuged at $12,000 \times \mathrm{g}$ for $5 \mathrm{~min}$.

\section{Acknowledgments}

We gratefully acknowledge Peter Lea for a critical reading of the manuscript. We are indebted to Antonietta Cirasuolo and Roberto Silva for their technical help. The antibodies to grapevine GDH were a generous gift from Professor Kaliopi Roubelakis-Angelakis.

\section{References}

[1] S. Proost, P. Pattyn, T. Gerats, Y. Van de Peer, Journey through the past: 150 million years of plant genome evolution, Plant J. 66 (2011) 58-65.

[2] R. De Smet, Y. Van de Peer, Redundancy and rewiring of genetic networks following genome-wide duplication events, Curr. Opin. Plant Biol. 15 (2012) $168-176$.

[3] F.J. Turano, S.S. Thakkar, T. Fang, J.M. Weisemann, Characterization and expression of $\mathrm{NAD}(\mathrm{H})$-dependent glutamate dehydrogenase genes in Arabidopsis, Plant Physiol. 113 (1997) 1329-1341.

[4] F. Dubois, T. Tercé-Laforgue, M.-B. Gonzalez-Moro, J.-M. Estavillo, R. Sangwan, A. Gallais, et al., Glutamate dehydrogenase in plants: is there a new story for an old enzyme? Plant Physiol. Biochem. 41 (2003) 565-576.

[5] A. Pavesi, A. Ficarelli, F. Tassi, F.M. Restivo, Cloning of two glutamate dehydrogenase cDNAs from Asparagus officinalis: sequence analysis and evolutionary implications, Genome 43 (2000) 306-316.

[6] F.M. Restivo, Molecular cloning of glutamate dehydrogenase genes of Nicotiana plumbaginifolia: structure analysis and regulation of their expression by physiological and stress conditions, Plant Sci. 166 (2004) 971-982.

[7] Y. Miyashita, A.G. Good, Glutamate deamination by glutamate dehydrogenase plays a central role in amino acid catabolism in plants, Plant Signaling Behav. 3 (2008) 842-843.

[8] J.-X. Fontaine, F. Saladino, C. Agrimonti, M. Bedu, T. Tercé-Laforgue, T. Tétu, et al., Control of the synthesis and subcellular targeting of the two GDH genes products in leaves and stems of Nicotiana plumbaginifolia and Arabidopsis thaliana, Plant Cell Physiol. 47 (2006) 410-418.

[9] D.S. Skopelitis, N.V. Paranychianakis, A. Kouvarakis, A. Spyros, E.G. Stephanou, K.A. Roubelakis-Angelakis, The isoenzyme 7 of tobacco NAD(H)-dependent glutamate dehydrogenase exhibits high deaminating and low aminating activities in vivo, Plant Physiol. 145 (2007) 1726-1734.

[10] M. Watanabe, Y. Itho, Y. Jo, K. Yasuda, K. Kamachi, Y. Watanabe, Redox and translational regulation of glutamate dehydrogenase $\alpha$ subunits in Brassica napus under wounding stress, Plant Sci. 172 (2007) 1182-1192.

[11] C. Masclaux-Daubresse, M. Reisdorf-Cren, K. Pageau, M. Lelandais, O. Grandjean, J. Kronenberger, et al., Glutamine synthetase-glutamate synthase pathway and glutamate dehydrogenase play distinct roles in the sink-source nitrogen cycle in tobacco, Plant Physiol. 140 (2006) 444-456.

[12] M.P. Purnell, J.R. Botella, Tobacco isoenzyme 1 of NAD(H)-dependent glutamate dehydrogenase catabolizes glutamate in vivo, Plant Physiol. 143 (2007) $530-539$.

[13] J.-X. Fontaine, T. Tercé-Laforgue, P. Armengaud, G. Clément, J.-P. Renou, S. Pelletier, et al., Characterization of a NADH-dependent glutamate dehydrogenase mutant of Arabidopsis demonstrates the key role of this enzyme in root carbon and nitrogen metabolism, Plant Cell 24 (2012) 4044-4065.

[14] J.-X. Fontaine, T. Tercé-Laforgue, S. Bouton, K. Pageau, P.J. Lea, F. Dubois, et al., Further insights into the isoenzyme composition and activity of glutamate dehydrogenase in Arabidopsis thaliana, Plant Signaling Behav. 8 (2013) 1-5.

[15] X. Qiu, W. Xie, X. Lian, Q. Zhang, Molecular analyses of the rice glutamate dehydrogenase gene family and their response to nitrogen and phosphorous deprivation, Plant Cell Rep. 28 (2009) 1115-1126.

[16] Y.-H. Lee, M. Tegeder, Selective expression of a novel high-affinity transport system for acidic and neutral amino acids in the tapetum cells of Arabidopsis flowers, Plant J. 40 (2004) 60-74.

[17] A. Schneidereit, J. Scholz-Starke, M. Buttner, Functional characterization and expression analyses of the glucose-specific AtSTP9 monosaccharide transporter in pollen of Arabidopsis, Plant Physiol. 133 (2003) 182-190.

[18] W. Schulze, E. Schulze, J. Stadler, H. Heilmeier, M. Stiit, H. Mooney, Growth and reproduction of Arabidopsis in relation to storage of starch and nitrate in the wild type and in starch-deficient and nitrate uptake deficient mutants, Plant Cell Environ. 17 (1994) 795-809.

[19] J.M. Alvarez, E.A. Vidal, R.A. Gutiérrez, Integration of local and systemic signaling pathways for plant N responses, Curr. Opin. Plant Biol. 15 (2012) $185-191$.

[20] G.M. Coruzzi, L. Zhou, Carbon and nitrogen sensing and signaling in plants: emerging "matrix effects", Curr. Opin. Plant Biol. 4 (2001) 247-253.

[21] Z.-L. Zheng, Carbon and nitrogen nutrient balance signaling in plants, Plant Signaling Behav. 4 (2009) 584-591.

[22] G. Krouk, S. Ruffel, R.A. Gutiérrez, A. Gojon, N.M. Crawford, G.M. Coruzzi, et al., A framework integrating plant growth with hormones and nutrients, Trends Plant Sci. 16 (2011) 178-182.

[23] H. Sakakibara, K. Takei, N. Hirose, Interactions between nitrogen and cytokinin in the regulation of metabolism and development, Trends Plant Sci. 11 (2006) 440-448.

[24] S. Ruffel, G. Krouk, D. Ristova, D. Shasha, K.D. Birnbaum, G.M. Coruzzi, Nitrogen economics of root foraging: transitive closure of the nitrate-cytokinin relay and distinct systemic signaling for N supply vs. demand, Proc. Natl. Acad. Sci. U. S. A. 108 (2011) 18524-18529.

[25] W.G. Brenner, G.A. Romanov, I. Köllmer, L. Bürkle, T. Schmülling, Immediateearly and delayed cytokinin response genes of Arabidopsis thaliana identified by genome-wide expression profiling reveal novel cytokinin-sensitive processes and suggest cytokinin action through transcriptional cascades, Plant J. 44 (2005) 314-333. 
[26] T. Kiba, T. Kudo, M. Kojima, H. Sakakibara, Hormonal control of nitrogen acquisition: roles of auxin, abscisic acid, and cytokinin, J. Exp. Bot. 62 (2011) 1399-1409.

[27] D. Igarashi, Y. Izumi, Y. Dokiya, K. Totsuka, E. Fukusaki, C. Ohsumi, Reproductive organs regulate leaf nitrogen metabolism mediated by cytokinin signal, Planta 229 (2009) 633-644.
[28] M.E. Schranz, M.A. Lysak, T. Mitchell-Olds, The ABC's of comparative genomics in the Brassicaceae: building blocks of crucifer genomes, Trends Plant Sci. 11 (2006) 535-542.

[29] C.A. Loulakakis, K.A. Roubelakis-Angelakis, Immunocharacterization of NADHglutamate dehydrogenase from Vitis vinifera L. Plant Physiol. 94 (1990) 109-113. 